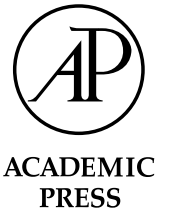

\title{
Kinetic behavior of Desulfovibrio gigas aldehyde oxidoreductase encapsulated in reverse micelles ${ }^{2 / 3}$
}

\author{
Susana L.A. Andrade, ${ }^{\text {a }}$ Carlos D. Brondino,,${ }^{\text {a,b,* }}$ Elvira O. Kamenskaya, ${ }^{c}$ \\ Andrey V. Levashov, ${ }^{c}$ and José J.G. Moura ${ }^{\mathrm{a}, *}$ \\ ${ }^{a}$ REQUIMTE/CQFB, Departamento de Química, Faculdade de Ciências e Tecnologia, Universidade Nova de Lisboa, Caparica 2829-516, Portugal \\ ${ }^{\mathrm{b}}$ Facultad de Bioquímica y Cs. Biológicas, Universidad Nacional del Litoral, CC 242, Santa Fe 3000, Argentina \\ ${ }^{\mathrm{c}}$ Division of Chemical Enzymology, Chemistry Department, Moscow State University, Moscow 119899, Russia
}

Received 27 June 2003

\begin{abstract}
We report the kinetic behavior of the enzyme aldehyde oxidoreductase (AOR) from the sulfate reducing bacterium Desulfovibrio gigas ( $D g$ ) encapsulated in reverse micelles of sodium bis-(2-ethylhexyl) sulfosuccinate in isooctane using benzaldehyde, octaldehyde, and decylaldehyde as substrates. $D g$ AOR is a $200-\mathrm{kDa}$ homodimeric protein that catalyzes the conversion of aldehydes to carboxylic acids. Ultrasedimentation analysis of $D g$ AOR-containing micelles showed the presence of $100-\mathrm{kDa}$ molecular weight species, confirming that the $D g$ AOR subunits can be dissociated. UV-visible spectra of encapsulated $D g$ AOR are indistinguishable from the enzyme spectrum in solution, suggesting that both protein fold and metal cofactor are kept intact upon encapsulation. The catalytic constant $\left(k_{\text {cat }}\right)$ profile as a function of the micelle size $W_{0}\left(W_{0}=\left[\mathrm{H}_{2} \mathrm{O}\right] /[\mathrm{AOT}]\right)$ using benzaldehyde as substrate showed two bell-shaped activity peaks at $W_{0}=20$ and 26. Furthermore, enzymatic activity for octaldehyde and decylaldehyde was detected only in reverse micelles. Like for the benzaldehyde kinetics, two peaks with both similar $k_{\text {cat }}$ values and $W_{0}$ positions were obtained. EPR studies using spin-labeled reverse micelles indicated that octaldehyde and benzaldehyde are intercalated in the micelle membrane. This suggests that, though $D g$ AOR is found in the cytoplasm of bacterial cells, the enzyme may catalyze the reaction of substrates incorporated into a cell membrane.
\end{abstract}

(c) 2003 Elsevier Inc. All rights reserved.

Keywords: Aldehyde oxidoreductase; Reverse micelles; Kinetic assay

In the living cell, enzymes function in a micro-heterogeneous environment, interacting with or being incorporated into different membrane structures. Even in the cytoplasm, water mainly plays a structural and functional role, far from being the dominant component as in a buffered laboratory assay. An in vitro system that tries to approach these in vivo conditions is the one that makes use of the self-organization and assembly of

\footnotetext{
Abbreviations: AOR, aldehyde oxidoreductase; Dg, Desulfovibrio gigas; DCPIP, 2,6-dichlorophenol indophenol; AOT, sodium bis(2-ethylhexyl) sulfosuccinate; $x$-DSA, $x$-doxyl stearic acid $(x=5,7$, or 10$) ; K_{\mathrm{m}}$, Michaelis-Menten constant; $V_{\max }$, maximal rate of the enzymatic reaction; $k_{\text {cat }}$, catalytic constant; EPR, electron paramagnetic resonance.

${ }^{*}$ Corresponding authors. Fax: +351-21-2948550 (J.J.G. Moura).

E-mail addresses: carlos.brondino@dq.fct.unl.pt (C.D. Brondino), jose.moura@dq.fct.unl.pt (J.J.G. Moura).
}

surfactant molecules in organic solvents to form reverse micelles [1-3]. The surfactant molecules (micelle membrane) separate and stabilize an aqueous inner core from the outer organic solvent in such a way that the hydrophobic parts are in contact with the non-polar bulk solution and the polar groups are facing towards the inner polar core. This polar part contains two welldifferentiated zones: the water molecules hydrating the surfactant polar heads (bound water) and those situated at the innermost part of the core (free water). Thus, hydrated reverse micelles possess up to four different microenvironments in which hydrophilic or hydrophobic molecules can partition according to their chemical affinities.

Several works on the catalytic properties of enzymes encapsulated in reverse micelles have been reported in the last years $[1,3,4]$. These studies showed that the 
biological activity is kept or even increased depending on the value of the parameter $W_{0}\left(W_{0}=\left[\mathrm{H}_{2} \mathrm{O}\right] /[\right.$ surfactant]), which is directly proportional to the micelle geometrical dimensions [5]. In some cases the catalytic constant of encapsulated enzymes as a function of $W_{0}$ reveals a bell-shaped peak in the case of monomeric proteins, whereas in the case of oligomeric enzymes there are as many peaks as the number of subunits $[6,7]$. Ultrasedimentation studies demonstrated that these catalytic constant peaks occur when there is a geometrical fit between the size of the enzyme subunit(s) and the radius of the micelle inner water core [8-10], indicating that reverse micelles can be used to check the subunit functionality of oligomeric proteins. Furthermore, because of the ability of reverse micelles to stabilize hydrophobic molecules, they can be advantageously used to study the catalysis of water-insoluble substrates, a goal impossible to achieve using conventional enzymatic assays in aqueous solutions.

We report here the catalytic properties of the enzyme aldehyde oxidoreductase (AOR) from the sulfate reducing bacterium Desulfovibrio gigas, encapsulated in reverse micelles of sodium bis-(2-ethylhexyl) sulfosuccinate (AOT) in isooctane. $D g$ AOR is a cytoplasmic enzyme capable of hydroxylation of several water-soluble aldehydes according to the reaction: $\mathrm{R}-\mathrm{COH}+\mathrm{H}_{2} \mathrm{O} \rightarrow$ $\mathrm{R}-\mathrm{COOH}+2 \mathrm{H}^{+}+2 \mathrm{e}^{-}$[11]. The protein is a $200-\mathrm{kDa}$ homodimer, each monomer having an active site composed of a molybdenum atom bound to a pterin unit (a molybdopterin cytosine dinucleotide) and two ironsulfur clusters that function as electron carriers [12]. We checked the catalytic properties of the $D g$ AOR monomer as well as the enzyme ability to catalyze the reactions of the water-insoluble octaldehyde and decylaldehyde. Changes in the polarity and fluidity of the reverse micelle membrane upon substrate addition were also analyzed.

\section{Materials and methods}

Chemicals. All chemical reagents were of analytical grade and used without any further purification. AOT (Sigma) was kept in a dry atmosphere under vacuum in order to minimize traces of water molecules. Fresh solutions of benzaldehyde (Sigma) were prepared in distilled water. Octaldehyde and decylaldehyde (Sigma) were diluted in isooctane (Aldrich). The spin probes $x$-DSA ( $x=5,7$, and 10) were purchased from Aldrich and dissolved in ethanol.

Enzyme purification. Dg AOR was purified up to electrophoretic grade according to published procedures [13]. Enzyme concentration was determined from its absorption at $460 \mathrm{~nm} \quad\left(\varepsilon_{460 \mathrm{~nm}}=20,000\right.$ $\left.\mathrm{M}^{-1} \mathrm{~cm}^{-1}\right)$.

Preparation of reverse micelles. AOT reverse micelles were prepared according to the injection method [14]. Stock solutions of AOT in isooctane were used when at least one day old to assure solution stability. To obtain reverse micelles with a given $W_{0}$, an aqueous volume $(v)$ was added to the AOT in isooctane solution according to $v(\mathrm{ml})=([\mathrm{AOT}] \times V(\mathrm{ml}) / 1000) \times 18 \times W_{0}$, where $V$ is the total volume of the organic solution and 18 is the molar volume of water $(\mathrm{ml} / \mathrm{mol})$. Immediately after addition of the aqueous components, the solution was vigorously shaken for a few seconds until it became transparent.

Determination of the aldehyde oxidoreductase activity. Enzymatic assays were carried out aerobically at $25^{\circ} \mathrm{C}$ in a quartz cell equipped with a magnetic stirrer device. UV-visible absorption spectra and kinetic assays were performed on a Shimadzu UV-2101 PC split-beam spectrophotometer. Conversion of aldehydes to the reaction product was determined by following the parallel reduction of DCPIP at $600 \mathrm{~nm}$ over time [15]. Protein solutions were prepared in $50 \mathrm{mM} \mathrm{im}$ idazole, $\mathrm{pH}$ 8.0. This buffer facilitates the protein encapsulation at low $W_{0}$ values [16] and contributes to the overall stabilization of the reverse micelle structure (see below in EPR results). The $\mathrm{pH}$ of the solution is a critical factor to stabilize the blue oxidized form of DCPIP in reverse micelles with $W_{0} \leqslant 15$.

Reactions were started by addition of substrate (or enzyme) after measuring the background stability of DCPIP in the reaction mixture. The initial rates were measured by calculating the slope of the tangent line at the initial stage of the progression curve for each reaction (lasting 20-150s or more, depending on the enzyme concentration) and taking into consideration the DCPIP molar absorption coefficient $\left(\varepsilon_{600 \mathrm{~nm}}=21,000 \mathrm{M}^{-1} \mathrm{~cm}^{-1}\right.$ in aqueous solution and $18,500 \mathrm{M}^{-1} \mathrm{~cm}^{-1}$ in $0.1 \mathrm{M}$ AOT reverse micelles - data not shown). Full kinetic assays were carried out for each $W_{0}$. Specific enzyme activity was found to be directly proportional to the enzyme concentration in the range $34-180 \mathrm{nM}$, while both substrate and DCPIP were kept constant at a saturating non-inhibiting amount (about 100 and $35 \mu \mathrm{M}$, respectively). The enzyme maximum velocity $\left(V_{\max }\right)$ and the Michaelis-Menten constant $\left(K_{\mathrm{m}}\right)$ were obtained from a Lineweaver-Burk linearization and reconfirmed by a non-linear regression analysis of the experimental data to the hyperbolic curve defined by a simple Michaelis-Menten equation [17]. Both the enzyme concentration, which is used to calculate the catalytic constant $\left(k_{\text {cat }}=V_{\max } /[\mathrm{AOR}]\right)$, and the amount of substrate added in each assay are referred to the total volume of the micelle solution $[18,19]$. All the kinetic assays were carried out under the same experimental conditions in order to compare the catalytic properties of the enzyme in both systems (reverse micelle and aqueous solutions).

Aldehyde partition in an isooctane/water biphasic system. In order to know the water solubility of benzaldehyde, octaldehyde, and decylaldehyde, increasing volumes of each aldehyde were added to a 1:1 mixture of Millipore water and isooctane. The organic phase (in the case of the water-insoluble aldehydes) was analyzed in a Lichrosorb RP18, $5 \mu \mathrm{m}$ (250-4) LKB column connected to an HPLC apparatus and elution was followed at $290 \mathrm{~nm}$. Eluent was isooctane (HPLC grade) with a linear flow of $1 \mathrm{ml} \mathrm{min}{ }^{-1}$. Extraction temperature and shaking time (without incubation) were the same as those in the kinetic assays. Water solubility was calculated considering the resulting peak areas. The experimental process to determine the benzaldehyde partition was basically the same, but the water phase was then analyzed. A mixture of $70 / 30 \%$ (v/v) acetonitrile and water was used as eluent. Upon continuous decrease in the initial water percentage, elution was followed at $250 \mathrm{~nm}$. Control assays were performed with the aldehydes solubilized in the phase under study. All the samples were analyzed in triplicate.

Ultrasedimentation analysis. The sedimentation coefficients of empty and protein-containing reverse micelles were measured at $25^{\circ} \mathrm{C}$ on a Beckman $\mathrm{E}$ analytical centrifuge equipped with a photoelectric scanning device with a monochromator and a multiplexor, using $12 \mathrm{~mm}$ bisector compartments and an An-G-Ti rotor at 20,000 rpm [7]. The scanning was carried out at 300 and $460 \mathrm{~nm}$. The sedimentation coefficients of empty and protein-containing micelles, and consequently the molecular weight of protein species dissolved in micelles, were calculated using conventional methods $[7,8]$.

EPR spectroscopy. EPR measurements at $9.7 \mathrm{GHz}$ (X-band) were performed on a Bruker EMX spectrometer equipped with a 
rectangular cavity (Model ER 4102ST) and $100 \mathrm{kHz}$ field modulation frequency. The spectra were acquired at room temperature $\left(25 \pm 1{ }^{\circ} \mathrm{C}\right)$ with a modulation amplitude of $1 \mathrm{G}$ and a microwave power of $2 \mathrm{~mW}$. Reverse micelle solutions of $W_{0}=20$ and 26 (without enzyme) were labeled by adding the spin probe to a final concentration of $54 \mu \mathrm{M}$. In these conditions, all the spin labels were incorporated into the micelle membrane with no detectable spinspin interactions.

Changes in the polarity and fluidity of the micelle membrane were evaluated upon addition of increasing amounts of aldehydes to 5DSA labeled micelles. EPR spectra of both 7-DSA and 10-DSA-labeled reverse micelles were indistinguishable from that of the probe in isooctane. The resemblance of the spectra of 7-DSA and 10-DSA in the reverse micelles to those of the probes in isooctane is probably due to the fact that carbons 7 and 10 are not in the micelle in view of the length of AOT. Thus, although these molecules are also incorporated in the micelle, the portion containing carbons 7 and 10 is immersed in the solvent. Membrane fluidity changes were estimated by measuring the order parameter according to $S=\left(A_{/ /}^{\prime}-A_{\perp}^{\prime}\right) /$ $\left[A_{z z}-\left(A_{x x}+A_{y y}\right) / 2\right]$, where $A_{\perp}^{\prime}$ and $A_{/ /}^{\prime}$ are the perpendicular and parallel components of the nitrogen hyperfine tensor $(S=1 / 2, I=1)$ (see Fig. 1) and $A_{x x, y y, z z}$ are the components in the rigid limit [20]. The hyperfine parameters $A_{\perp}^{\prime}$ and $A_{/ /}^{\prime}$ were measured from the experimental spectra as shown in Fig. 1 and $A_{x x, y y, z z}$ were obtained from [20]. The larger the $S$, the higher the order of the micelle membrane [20]. Variations in the micro polarity of the spin label environment were estimated by means of the isotropic hyperfine factor, according to $a_{0}=\left(A^{\prime}, 2 A_{\perp}^{\prime}\right) / 3[20]$. The larger the $a_{0}$, the higher the polarity of the probe environment [21,22]. No corrections for polarity changes in the $S$ parameter were taken into consideration [20] because the isotropic hyperfine parameter was constant within the experimental error.

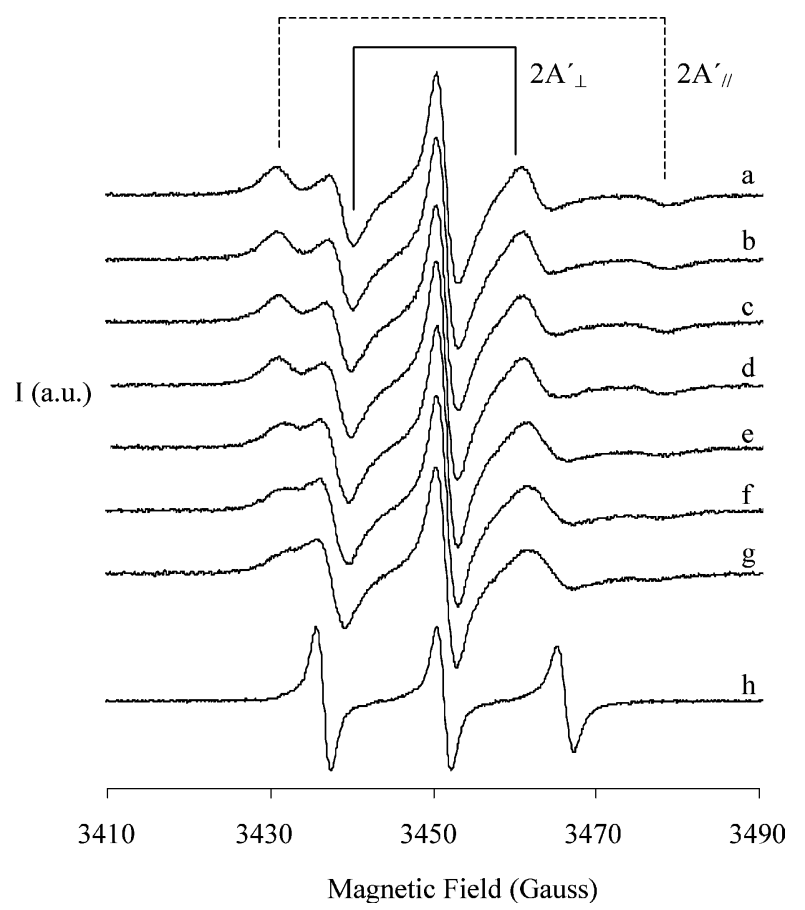

Fig. 1. EPR spectra of 5-DSA spin labeled 0.1 M AOT reverse micelles with $W_{0}=26$. (a) Reverse micelle solution without aldehyde, (b-g) upon increasing additions of octaldehyde, and (h) spectrum of the free spin probe in isooctane. Final aldehyde concentrations were: (b) $21 \mathrm{mM}$; (c) $43 \mathrm{mM}$; (d) $107 \mathrm{mM}$; (e) $213 \mathrm{mM}$; (f) $320 \mathrm{mM}$; and (g) $426 \mathrm{mM}$.

\section{Results and discussion}

\section{Sedimentation analysis}

Sedimentation studies performed on empty and $D g$ AOR-containing reverse micelles in the hydration range of $W_{0}=10$ to 35 showed only the presence of protein molecules with a molecular weight of $100 \mathrm{kDa}$. This result indicates that the dimeric $D g$ AOR can dissociate into stable monomers when encapsulated in reverse micelles. Moreover, the UV-visible spectra of $D g$ AOR encapsulated in $0.1 \mathrm{M}$ AOT reverse micelles in the same hydration range ( $W_{0}=10$ to 35$)$ are similar to the spectrum of the protein in aqueous solution, suggesting that protein molecular properties such as folding and structure of the metal cofactors are kept upon encapsulation.

\section{Localization of aldehyde molecules in reverse micelles}

To determine how the substrate molecules are distributed in reverse micelles, we evaluated the isooctane/ water partition coefficients for the different substrates, as well as the influence of the aldehyde molecule on the micelle membrane using the spin labeling technique. The determination of the partition coefficients $(P=$ $[\text { aldehyde }]_{\text {isooctane }} /[\text { aldehyde }]_{\mathrm{H}_{2} \mathrm{O}}$ ) for benzaldehyde, octaldehyde, and decylaldehyde yielded $0.56,1.73$, and 2.02 (values in $\log P$ ), respectively. According to these values, only about $1 \%, 2 \%$, and $22 \%$ of decylaldehyde, octaldehyde, and benzaldehyde, respectively, are present in the aqueous phase, revealing that the long chain aldehydes are practically insoluble in water.

Although such measurements were reported to give a good estimation of the partition of molecules between the organic and the aqueous pseudophases in a reverse micelle system [19], the micelle membrane, as seen below, may also compete with the other pseudophases to accommodate the aldehyde molecules. Fig. 1 shows the EPR spectra of 5-DSA-labeled reverse micelles without (a) and upon octaldehyde addition (b-g) together with the spectrum of the probe in isooctane $(\mathrm{h})$. Comparison of spectra $(\mathrm{a}-\mathrm{g})$ with spectrum $(\mathrm{h})$ indicate that the spin probe is located in the reverse micelle membrane, and therefore, 5-DSA constitutes a good marker to evaluate changes experimented by the micelle membrane. As shown in Fig. 1, the EPR spectrum modifications indicate that aldehyde molecules perturb the reverse micelle membrane. These alterations are analyzed in Fig. 2, which shows the evolution of the order parameter $(S)$ upon octaldehyde additions to $0.1 \mathrm{M}$ AOT reverse micelle solutions with $W_{0}=26$. The inverse linear relations obtained for the $S$ parameter clearly indicates that at least part of the aldehyde molecules are located within the reverse micelle membrane increasing its fluidity. On the contrary, no significant changes were detected for 


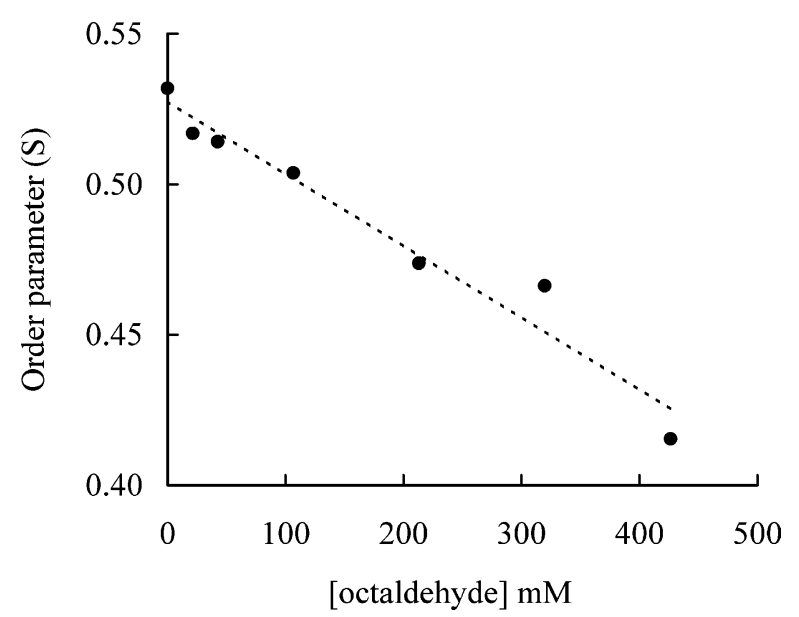

Fig. 2. Evolution of the order parameter $(S)$ as a function of octaldehyde additions to 5-DSA spin labeled 0.1 M AOT reverse micelles with $W_{0}=26 . S$ values were obtained from the spectra shown in Fig. 1.

the polarity parameter, which was found to remain constant within the experimental error $\left(a_{0}=14.9 \pm\right.$ $0.1 \mathrm{G})$. Similar results were obtained at $W_{0}=20$ and for decylaldehyde at $W_{0}=20$ and 26 (data not shown). Although water-insoluble aldehydes strongly affect the membrane fluidity, the absence of alterations in the polarity suggests that the water does not penetrate into the reverse micelle membrane upon aldehyde addition.

In contrast, identical studies carried out with benzaldehyde resulted in non-detectable fluidity alterations, indicating that benzaldehyde is not located in the micelle membrane. Therefore, it should be either in the water core or close to the membrane-water interface.

The integrity of the 5-DSA-labeled micelle membrane using either water or imidazole buffer was also checked for $0.1 \mathrm{M}$ AOT reverse micelles with $W_{0}=20$. Imidazolecontaining reverse micelles $\left(S=0.517 \pm 0.004, a_{0}=\right.$ $14.8 \pm 0.1 \mathrm{G})$ are more ordered and less polar than the water-containing ones $\left(S=0.491 \pm 0.007, a_{0}=15.3 \pm\right.$ $0.1 \mathrm{G})$. Thus, under the tested conditions, reverse micelles filled with water are less ordered than those filled with imidazole buffer.

\section{Benzaldehyde catalysis}

As observed before [15], the as-purified enzyme displayed a simple Michaelis-Menten kinetics for the benzaldehyde:DCPIP oxidoreductase activity in aqueous solution. The parameter $k_{\text {cat }}$ in $50 \mathrm{mM}$ imidazole buffer at $\mathrm{pH} 8.0$ is given in Table 1. No substrate inhibition was observed for the tested concentration range (up to $\sim 0.3 \mathrm{mM}$ ).

Fig. 3A shows the resulting kinetic profiles of $D g$ AOR encapsulated in a $0.1 \mathrm{M}$ AOT solution as a function of $W_{0}$. As seen in this figure, two bell-shaped peaks with similar $k_{\text {cat }}$ values were obtained at $W_{0} \sim 20$
Table 1

Kinetic parameters of $D g$ AOR in aqueous solution and in AOT reverse micelles with different hydration degrees $\left(W_{0}\right)$

\begin{tabular}{llc}
\hline Substrate & $W_{0}$ & $k_{\text {cat }}\left(\mathrm{s}^{-1}\right)$ \\
\hline \multirow{3}{*}{ Benzaldehyde } & Solution & $1.35 \pm 0.04$ \\
& 20 & $1.3 \pm 0.1$ \\
Octaldehyde & 26 & $1.25 \pm 0.05$ \\
& 20 & $0.8 \pm 0.1$ \\
Decylaldehyde & 26 & $1.2 \pm 0.1$ \\
& 20 & $1.19 \pm 0.07$ \\
& 26 & $1.22 \pm 0.07$ \\
\hline
\end{tabular}

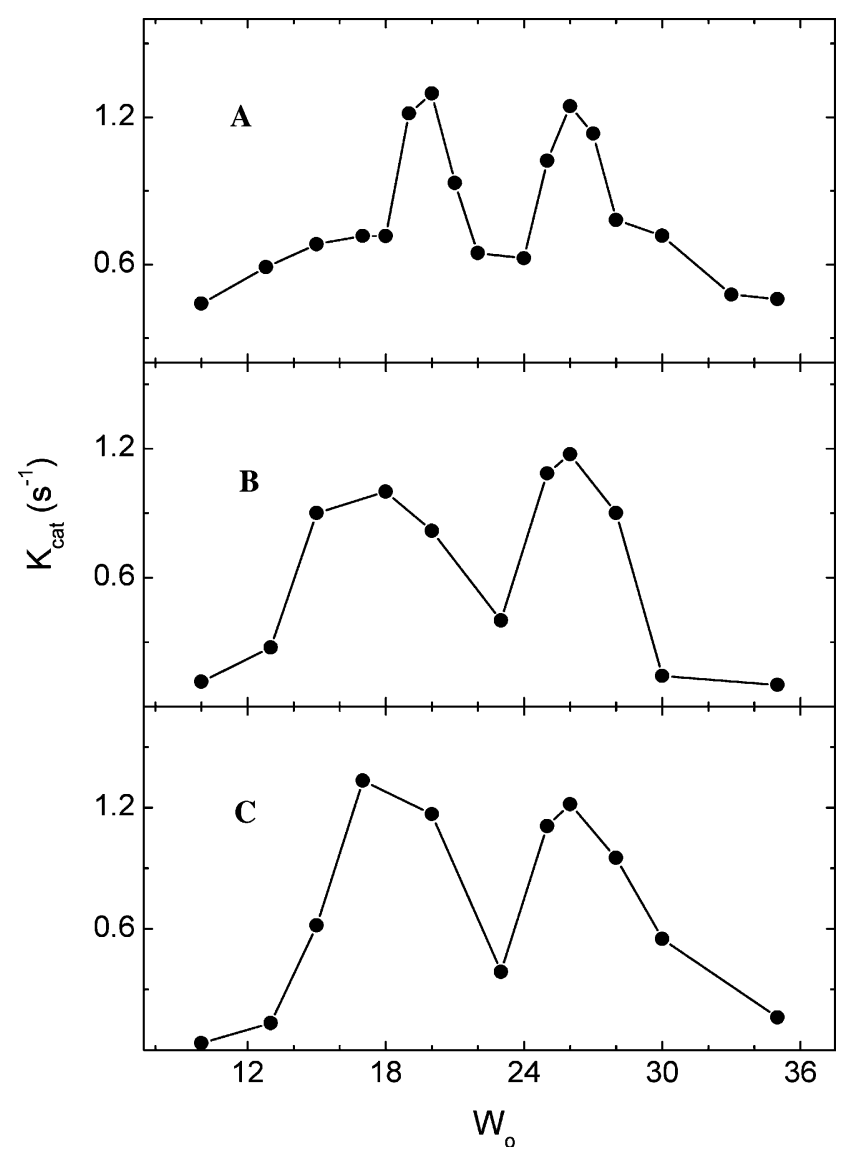

Fig. 3. Profiles of the catalytic constant as a function of the hydration degree $\left(W_{0}=\left[\mathrm{H}_{2} \mathrm{O}\right] /[\mathrm{AOT}]\right)$ for $D g$ AOR encapsulated in $0.1 \mathrm{M}$ AOT reverse micelles. Activity assays were performed at $25^{\circ} \mathrm{C}$ using $30 \mu \mathrm{M}$ DCPIP and 20-52 nM enzyme in $50 \mathrm{mM}$ imidazole buffer, $\mathrm{pH}$ 8.0. The substrates used were (A) benzaldehyde, (B) octaldehyde, and (C) decylaldehyde.

and $\sim 26$. Considering the fact that the protein has approximately a globular shape [23], peaks of enzymatic activity should occur when the size of the reverse micelle inner core matches the protein size, that is when $r_{\mathrm{m}} \sim r_{\mathrm{p}}$, where $r_{\mathrm{m}}$ is the inner cavity micelle radius and $r_{\mathrm{p}}$ is the mean protein radius. The micelle 
inner cavity radius is given by $r_{\mathrm{m}}(\mathrm{A})=4+1.5 W_{0}$ [9], whereas the mean protein radius may be calculated with the equation: $r_{\mathrm{p}}(\AA)=0.7\left(M_{\mathrm{r}}\right)^{1 / 3}$, where $M_{\mathrm{r}}$ represents the protein molecular weight $(\mathrm{g} / \mathrm{mol})$ [24,25]. Both equations predict activity peaks at $W_{0} \sim 19$ for the monomer and $W_{0} \sim 25$ for the dimer, in good agreement with the experimental data (Fig. 3A). This indicates that monomeric species are responsible for the activity peak at $W_{0} \sim 20$, whereas dimeric species produce the peak at $W_{0} \sim 26$. This proves the hypothesis derived from structural data [23] that $D g$ AOR monomers may act independently.

Interestingly, whereas the kinetic studies confirmed the presence of $D g$ AOR dimeric form at $W_{0} \sim 26$, the sedimentation studies could only detect monomers. These apparently contradictory results may be tentatively explained considering a monomer-dimer dynamical equilibrium. Our current hypothesis is that this equilibrium would tend towards the dimer in aqueous solution, as all the previous biochemical and molecular properties of $D g$ AOR in solution have shown [13]. On the contrary, in reverse micelles the predominant form appears to be the monomer, as it was concluded by our sedimentation analysis. The detection of an activity peak at $W_{0} \sim 26$ (the theoretical catalytic activity maximum for an AOR dimer) indicates that this equilibrium in reverse micelles is shifted under turnover conditions (Table 1). Other substrate-depending effects have been previously observed in reverse micelles. For example, in the case of lysozyme encapsulated in reverse micelles, the substrate was found to regulate the equilibrium between denatured and native enzyme towards the native conformation [26].

\section{Octaldehyde and decylaldehyde catalysis}

$D g$ AOR enzymatic assays carried out in the aqueous fraction that resulted from partition experiments carried out with octaldehyde and decylaldehyde in 1:1 isooctane/water mixtures showed no activity towards the respective aldehyde. However, as it is shown, their catalysis could be measured in reverse micelles. As seen in Figs. 3B and C, the $k_{\text {cat }}$ evolution as a function of $W_{0}$ for the water-insoluble aldehydes also showed two bell-shaped peaks with $k_{\text {cat }}$ values and $W_{0}$ positions similar to those observed for the benzaldehyde assay (Table 1). Further comparison of Fig. $3 \mathrm{~A}$ with $\mathrm{B}$ and $\mathrm{C}$ reveals broader $k_{\text {cat }}$ peaks for the water-insoluble aldehydes. As it was concluded from the partition experiments above, these long chain aldehydes are found in the micelle membrane. The consequences of this over the micelle properties may eventually explain the enzyme stabilization and consequently broader activity peaks over a wider range of $W_{0}$.

\section{Conclusions}

As a system that mimics a cell environment, reverse micelles offer new possibilities of studying enzymes and bioconversions of non-polar compounds [27]. The present study shows the enzymatic ability of $D g$ AOR encapsulated in AOT-water-isooctane reverse micelles. Taking advantage of this multiphase system, we were able to demonstrate that $D g$ AOR monomers can be separated under non-denaturing conditions in a stable and active form. Considering previous studies that have shown that $D g$ AOR can catalyze the reactions of a broad range of aldehydes, we used reverse micelles to show for the first time the ability of this enzyme to catalyze the reactions of water-insoluble aldehydes. These aldehydes are located in the reverse micelle membrane, which suggests that $D g$ AOR, though localized in the cytoplasm, might catalyze reduction of substrates incorporated into cell membranes.

\section{Acknowledgments}

This work was supported by Fundação para a Ciência e Tecnologia POCTI/BME/36152/99 (Portugal) and CAI+D-UNL (Argentina). We thank Prof. Natalia L. Klyachko for help with the ultrasedimentation experiments.

\section{References}

[1] K. Martinek, A.V. Levashov, N.L. Klyachko, Y.L. Khmelnitski, I.V. Berezin, Micellar enzymology, Eur. J. Biochem. 155 (1986) 453-468.

[2] A. Erjomin, D. Metelitza, Catalysis by hemoproteins and their structural organization in reverse micelles of surfactants in octane, Biochim. Biophys. Acta 732 (1983) 377-386.

[3] R. Bru, A. Sanchez-Ferrer, F. Garcia-Carmona, Kinetic models in reverse micelles, Biochem. J. 310 (1995) 721-739.

[4] Y.L. Khmelnitsky, A.V. Kabanov, N.L. Klyachko, A.V. Levashov, K. Martinek, in: M.P. Pileni (Ed.), Structure and Reactivity in Reverse Micelles, Elsevier, Amsterdam, 1989, p. 379.

[5] J.D. Nicholson, J.H.R. Clarke, in: K.L. Mittal, B. Lindman (Eds.), Surfactants in Solution, Plenum Press, New York, 1984, pp. 1663-1674.

[6] A.V. Levashov, R.V. Rarity, K. Martinek, N.L. Klyachko, Artificial glycosylated alpha-chymotrypsin in reverse micelles of aerosol OT in octane - a new approach to elucidation of the role of carbohydrate moieties in glycoproteins, FEBS Lett. 336 (1993) 385-388.

[7] K. Martinek, N.L. Klyachko, A.V. Kabanov, Y.L. Khmelnitsky, A.V. Levashov, Micellar enzymology_its relations to membranology, Biochim. Biophys. Acta 981 (1989) 161-172.

[8] A.V. Kabanov, S.N. Nametkin, G.N. Evtushenko, N.N. Chernov, N.L. Klyachko, A.V. Levashov, K. Martinek, A new strategy for the study of oligomeric enzymes - gamma-glutamyltransferase in reverse micelles of surfactants in organic solvents, Biochim. Biophys. Acta 996 (1989) 147-152.

[9] A.V. Kabanov, N.L. Klyachko, S.N. Nametkin, S. Merker, A.V. Zaroza, V.I. Bunik, M.V. Ivanov, A.V. Levashov, Engineering of functional supramacromolecular complexes of proteins (enzymes) 
using reversed micelles as matrix microreactors, Protein Eng. 4 (1991) 1009-1017.

[10] A.V. Levashov, Y.L. Khmelnitsky, N.L. Klyachko, V.Y. Chernyak, K. Martinek, Enzymes entrapped into reverse micelles in organic-solvents. Sedimentation analysis of the protein-aerosol OT- $\mathrm{H}_{2} \mathrm{O}$-octane system, J. Colloid Interf. Sci. 88 (1981) 444457.

[11] R. Hille, The mononuclear molybdenum enzymes, Chem. Rev. 96 (1996) 2757-2816.

[12] M.J. Romão, J. Knäblein, R. Huber, J.J.G. Moura, Structure and function of molybdopterin containing enzymes, Prog. Biophys. Molec. Biol. 68 (1997) 121-144.

[13] J.J.G. Moura, A.V. Xavier, M. Brushi, J. LeGall, D.O. Hall, R. Cammack, Molybdenum-containing iron-sulfur protein from Desulfovibrio gigas, Biochem. Biophys. Res. Commun. 72 (1976) 782-789.

[14] K. Martinek, A.V. Levashov, N.L. Klyachko, I.V. Berezin, Catalysis by water soluble enzymes in organic-solvents. Stabilization of enzymes against denaturation (inactivation) when they are included in reverse micelles of surface-active substance, Dokl. Akad. Nauk. 236 (1977) 920-925.

[15] B.A.S. Barata, J. LeGall, J.J.G. Moura, Aldehyde oxidoreductase activity in Desulfovibrio gigas - In vitro reconstitution of an electron transfer chain from aldehydes to the production of molecular hydrogen, Biochemistry 32 (1993) 11559-11568.

[16] S.L. Andrade, E.O. Kamenskaya, A.V. Levashov, J.J.G. Moura, Encapsulation of flavodoxin in reverse micelles, Biochem. Biophys. Res. Commun. 234 (1997) 651-654.

[17] A. Cornish-Bowden, Fundamentals of Enzyme Kinetics, Portland Press Ltd, London, 1981.

[18] A.V. Kabanov, A.V. Levashov, N.L. Klyachko, S.N. Namyotkin, A.V. Pshezhetsky, Enzymes entrapped in reverse micelles of surfactants in organic-solvents. A theoretical treatment of the catalytic activity regulation, J. Theor. Biol. 133 (1988) 327343.
[19] Y.L. Khmelnitsky, I.N. Neverova, V.I. Polyakov, V.Y. Grinberg, A.V. Levashov, K. Martinek, Kinetic theory of enzymaticreactions in reverse micellar systems. Applications of the pseudophase approach for partitioning substrates, Eur. J. Biochem. 190 (1990) 155-159.

[20] S. Schreier, C.F. Polnaszek, I.C.P. Smith, Spin labels in membranes. Problems in practice, Biochim. Biophys. Acta 515 (1978) 375-436.

[21] J. Harris, T.J. Power, A.L. Bieber, A. Watts, An electron-spin resonance spin-label study of the interaction of purified mojave toxin with synaptosomal membranes from rat-brain, Eur. J. Biochem. 131 (1983) 559-565.

[22] P. Baglioni, E. Ferroni, G. Martini, M.F. Ottaviani, Micellar solutions of sulfate surfactants studied by electron-spin-resonance of nitroxide radicals. 2. Use of C-8, C-12 and C-16 derivatives of piperidinyl-1-oxy, J. Phys. Chem. 88 (1984) 5107-5113.

[23] M.J. Romão, M. Archer, I. Moura, J.J.G. Moura, J. LeGall, R. Engh, M. Schneider, R. Huber, Crystal structure of the xanthine oxidase-related aldehyde oxidoreductase from D. gigas, Science 270 (1995) 1170-1176.

[24] A.V. Levashov, in: I.V. Berezin (Ed.), Chemical and Enzyme Reactions in Surfactants in Solution, VINITI Publishers, Moscow, 1987.

[25] Y.E. Shapiro, V.Y. Gorbatyuk, A.V. Levashov, N.L. Klyachko, Segmental mobility of molecules in the reversed micelle of aerosol OT in $N$-octane containing alpha-chymotrypsin and albumin, Biol. Mem. 7 (1994) 277-290.

[26] B. Steinmann, H. Jakle, P.L. Luisi, A comparative study of lysozyme, conformation in various reverse micellar systems, Biopolymers 25 (1986) 1133-1156.

[27] R. Hilhorst, C. Laane, C. Veeger, Enzymatic conversion of apolar compounds in inorganic media using an NADH-regenerating system and dihydrogen as reductant, FEBS Lett. 159 (1983) 225228. 